\title{
Large-Eddy Simulation of the Interaction of a Jet with a Wing
}

\author{
Maksym Bondarenko, ${ }^{*}$ Zhiwei $\mathrm{Hu}^{\dagger}$ and Xin Zhang ${ }^{\ddagger}$ \\ University of Southampton
}

\begin{abstract}
This paper presents progresses made on aircraft installation effects using numerical methods under WP 3.2 of SYMPHONY, a project supported by Technology Strategy Board, UK. Large-eddy simulations (LES) were performed for turbulent flow around a wing under the influence from engine jet flow by solving the compressible Navier-Stokes equations using an in-horse high-order finite difference code. Simulations were performed for jet under both a clean wing and the wing at high-lift configuration. Installation effects on both the jet and the wing are analysed by comparing with LES results performed for three baseline cases: jet along, clean wing along and the wing in high-lift configuration. It is found that the flow is two-dimensional near the leading edge of the wing. Further downstream three-dimensional flow features are developed. Interaction with vortical jet stream accelerates developments of the flow underneath the wing. Stronger turbulent structures are seen within the jet shear layer near the wing and their interaction with the wing causes surface pressure fluctuations, which results in increased radiated noise. Interaction with the jet causes a reduction in lift for the clean wing, however the contribution from the flap is increased when the wing is in high-lift configuration. For the current geometry the jet stream does not hit the clean wing, and it is shifted towards the wing by a small angle (one degree) due to low pressure region under the wing. When the flap is deployed, jet stream hits the flap and is deflected away from the wing.
\end{abstract}

\section{Nomenclature}

C Wing chord length

$c_{p} \quad$ Coefficient of pressure

$c_{\infty} \quad$ Sound speed

$C_{L} \quad$ Lift coefficient

$d_{t} \quad$ Time step

$D_{j} \quad$ Jet nozzle exit diameter

$L_{x} \quad$ Computational domain size in the streamwise $(x)$ direction

$L_{y} \quad$ Computational domain size in the spanwise $(y)$ direction

$L_{z} \quad$ Computation al domain size in the crossflow $(z)$ direction

$M_{j} \quad$ Jet Mach number

$M_{\infty} \quad$ Flight stream Mach number

$p \quad$ Pressure

$Q \quad$ Second invariant of velocity gradient tensors

$R e_{j} \quad$ Reynolds number based jet centerline velocity and diameter

$u, v, w$ Velocity components in Cartesian coordinates

$x, y, z$ Cartesian coordinates

$\rho \quad$ Density

${ }^{*}$ Research Assistant, Faculty of Engineering and the Environment.

${ }^{\dagger}$ Lecturer, Faculty of Engineering and the Environment, AIAA Member.

$\ddagger$ Airbus Professor of Aircraft Engineering, Faculty of Engineering and the Environment, Associate Fellow AIAA. 


\section{Introduction}

Interactions between aero-engines and the wing have strong influence on aeroplane aerodynamics and its noise impact on the environment. It is well known that engine position changes its performance. Engines on modern large commercial aircraft are often installed underneath the wing through nacelles. This configuration provides fresh air for the engine and also has other aerodynamic and structural benefits. However the fact that engines are in the close vicinity of the wing causes interaction between the jet stream and flow around the wing, which influences performances of both the wing and the engine, a phenomenon known as the installation effect, which has become a big concern for aerospace industry.

Aircraft noise is mainly generated by engines, high-lift devices and landing gears. To achieve aircraft reductions, it is necessary to reduce noise from all components that contribute significantly to the total noise and noise due to the interactions. For model aero-engines, noise is generated when the high speed flow of jet stream mixes with ambient fluid and due to interactions of vortical structures in the jet. When the engine is installed underneath the wing, jet flow also interacts with the wing.

Noise generation by the jet interacting with airframe, such as high-lift devices, is a fundamental problem affecting the design and performance of aircraft. Understanding of the installation effect would help in design more efficient and more environmental friendly aircraft. Flow mechanism and physics for noise generation by individual components have been studied extensively, using both experimental and numerical methods. However interactions between components are much more complicated. Both fly-over and wind tunnel tests have identified the influence on far field noise from interaction between different components. However the underlying mechanisms are still open questions and need further investigations.

Experiments have been carried out trying to quantify the characteristics and intensity of installation noise 1-5. Most of these experiments measured noise from jet stream alone and when the jet was placed near an aircraft wing model inside an anechoic chamber. Bhat ${ }^{6}$ reviewed studies on installation noise of wing-flap settings and jet engine. It was found that noise increases monotonically with increasing flap deflection, which was identified as the strongest effect on the installation noise. Also it was shown that pitching the nozzle up toward the wing increases the noise levels, while pitching the nozzle away from the wing has little benefit. Existing work on installation effect lack detailed flow information which makes it difficult to identify the underlying physics.

Due to limitation in computational power, previous numerical studies have not been able to consider aircraft installation effects. This paper performs large-eddy simulations (LES) for turbulent jet with the nozzle included, and more importantly underneath a wing model, to study jet/wing interaction. Current work is perform under WP 3.2 of SYMPHONY, a project supported by Technology Strategy Board, UK, with many aerospace industries, such as Airbus, Rolls-Royce and QinetiQ involved. High fidelity time-accurate numerical tools are used to investigate aerodynamics and noise due to the interaction of a jet with a wing. Simulations are performed for turbulent flow around a model wing with a constant span, under the influence of a jet. Five different jet/wing configurations were studied: jet with flight stream, clean wing, jet interacting with a clean wing, high-lift wing with flap deployed and jet interacting with high-lift wing. The aims is to investigate the aerodynamics due to the interaction of a jet with the wing and to understand the flow physics and noise generation mechanisms due to the jet/wing interaction.

\section{Numerical approach}

All simulations in this work are performed using an in-house high-order finite difference code SotonCAA, which can perform LES or detached-eddy simulations on block-structured grids to solve the unsteady compressible Navier-Stokes equations. SotonCAA uses high-order numerical schemes for both spatial discretization and time marching ${ }^{7}$. Time accurate simulations can be performed for unsteady flow. The code structure is based on a series of modules, in order to maximise the maintainability and expansion possibilities. A benefit of this structure is the convenience to implement new schemes, which can evolve easily over time. The disadvantage of this is that the code has grown rather complex with increasing number of subroutines.

The pre-factored, 6th order compact finite-difference scheme proposed by Hixon ${ }^{7}$ and later optimized by Ashcroft, et al ${ }^{8}$ is employed for the current simulations in preference to improve wavenumber resolutions for better dispersion characteristics, which is perferred for acoustic study. Finite difference filtering schemes are optional with 2nd-order, 6th-order and 10th-order central schemes coupled with biased schemes of reduced

order. Time integration, uses a low storage, low dispersion and dissipation Runge-Kutta (LDDRK) ${ }^{9}$ scheme 
which is 4th-order accurate 4-6 stage explicit scheme. Recently, an implicit 2nd-order time-accurate LU-SGS method ${ }^{10}$ has been introduced into SotonCAA by Ma, et al ${ }^{11}$. There are several selectable non-reflecting boundary conditions implemented in SotonCAA in order to perform acoustic calculations. As mentioned before, buffer zone schemes are preferred due to its excellent performance with non-linear flows and the easy way of implementation. In SotonCAA, structured grids are required for use of numerical calculation in order to obtain high-order accurate solutions. In order to improve the numerical stability in multi-block junctions, a characteristic interface condition proposed by Kim and Lee ${ }^{12}$ is also implemented. Turbulence models are selectable and treated in a modular manner so that additional models are easy to be added on. At the moment, SotonCAA turbulence models include Spallart-Allmaras (S-A) ${ }^{13}$, LES ${ }^{14}$, and detached-eddy simulation ${ }^{15}$.

Sound radiation to far field can be performed in SotonCAA through integration methods, such as the FW-H method ${ }^{16}$, and propagation by solving the linearized Euler equations or the acoustic perturbation equations ${ }^{16}$. SotonCAA is fully-parallelized using message-passing interface, and is portable to different plan form. In order to maintain a capacity for aeroacoustic noise prediction the SotonCAA code uses high order temporal and spatial schemes to keep wave dissipation and dispersion low. The code has several highorder finite-difference schemes available for spatial derivative calculations. A characteristics-based block interface treatment ${ }^{17}$ is used in order to avoid the discontinuity problems associated with the high-order finite difference schemes. It was demonstrated that a multi-block structured grid combined with high-order finite differencing schemes and a novel block-interface condition leads to reliable aeroacoustic solutions in such a highly complex geometry.

The solver was also required to have parallel capability so that large scale simulations could be performed on the high performance computing cluster, Iridis3, at the University of Southampton. The Iridis 3 HPC cluster includes 1008 8-core compute nodes (Intel Nehalem $2.26 \mathrm{GHz}$ ) with a minimum 24GB of memory per nod, a fast infiniband network for parallel communication, and a $100 \mathrm{~TB}$ of storage with high-performance GPFS file system.

\section{Mesh generation and simulation setup}

Five configurations were simulated in this study. Details of the computational domains and mesh sizes for all cases are given in Table 1.

Table 1: The computational domains and meshes for all cases.

\begin{tabular}{rrrrrr}
\hline \hline Cases & $L_{x}$ & $L_{y}$ & $L_{z}$ & Blocks & Grid points \\
\hline Jet & $25 D_{j}$ & $16 D_{j}$ & $16 D_{j}$ & 432 & $9.06 \mathrm{M}$ \\
Clean Wing & $15.7 C$ & $8.8 C$ & $3.4 C$ & 384 & $6.7 \mathrm{M}$ \\
Jet/Clean Wing & $25 D_{j}$ & $16 D_{j}$ & $3.5 D_{j}$ & 1,664 & $28.9 \mathrm{M}$ \\
Flap-deployed Wing & $17.7 C$ & $8.8 C$ & $3.4 C$ & 1106 & $22.2 \mathrm{M}$ \\
Jet/Flap-deployed Wing & $31.75 D_{j}$ & $18 D_{j}$ & $5.6 D_{j}$ & 1,642 & $40 \mathrm{M}$ \\
\hline
\end{tabular}

The computational domain used for the jet interacting with a clean wing case (jet/clean wing) is sketched in Fig. 1 with the model. The computational domain extends $25 D_{j}$ in the downstream direction, where $D_{j}$ is the nozzle exit diameter. A pipe is attached to the nozzle and extends $20 D_{j}$ upstream to help obtain a fully-developed turbulent flow at the jet inlet. The domain extends $8 D_{j}$ to both directions above and below the jet centerline. The spanwise box length is $3.5 D_{j}$. Due to the complex nature of the geometry for the nozzle/wing case, a total of structure 1,664 blocks are used to generate a fully-structured grid for this case. Grid topology of the multi-block structured grid is shown in Fig. 2. The total number of grid points used for this case is $28,894,264$. Care was taken to achieve high resolution in the near wall area and between the jet and the wing. Fig. 3 shows some details of the surface grids for the nozzle/wing case.

The geometry for jet interacting with the wing when the flap is deployed (jet/flap-deployed wing) is very complex. To implement a fully structured mesh for this case requires a huge number of grid points to resolve the nozzle and wing boundary layers for the Reynolds number being studied. The computational domain extends up to $31.75 D_{j}$ in the downstream direction, and $9 D_{j}$ above and below the jet centerline in the cross-flow direction. The spanwise computational domain size is $5.6 D_{j}$. The same as for all cases involving 
the jet, a pipe is connected in front of the nozzle to help the jet developing, the length of the pipe for this case is $16.6 D_{j}$. In the streamwise direction, the physical domain ends at $x=25 D_{j}$. The remaining region, from $x=25 D_{j}$ to $x=31.75 D_{j}$, is used as a sponge region to dissipate the fluctuations from the vortical flow from the jet and the separated flow behind the wing before they reach the outflow boundary to minimize reflection.

This case involves three separate solid elements, the nozzle, wing and the flap, making the geometry very complex. The surface grid topology for the three parts is shown in Fig. 4. Details of the surface mesh are provided in Fig. 5. A block structured grid is generated to ensure a high quality transition between the boundary layer mesh and the volume mesh. This took a large amount of effort due to the curvature and complexity of the geometry. The aim was to provide LES regions of the Wow Veld isotropic cells for efficiency and high resolution of small scales. Due to the high complexity around the nozzle/wing region a lot of work was undertaken to fill these regions with structured cells as necessary for LES. The final mesh comprises of 1,642 blocks and approximately 40 million cells.

LES was performed at a jet exit Mach number of 0.75 and a flight stream Mach number of 0.29 using the Smagorinski model. Reynolds number based on jet diameter and centreline flow conditions is $R e_{j}=$ $6,753,865$. Velocity inlet and pressure outlet boundary conditions were specified along the inflow and outflow far-field faces respectively. Symmetry boundary conditions were assigned to the remaining far-field boundaries and no-slip wall boundary conditions enforcing zero pressure gradients were assigned to the wing surface.

The LES simulation was performed on a total of 512 processors and ran for a total of 400,000 iterations. Each time step took approximately 10 seconds to complete leading to a total of 2500 CPU hours in this configuration in order to complete the simulation. Statistics are collected after the flow is deemed fully developed. Sampling of the data was then performed so that the mean flow results and the flow statistics could be obtained. Time-averaging of the flow was then performed for 150,000 time steps to calculate turbulence statistics.

\section{Installation effect on aerodynamics}

\section{A. Jet interacting with a clean wing}

Simulations were first run for a clean wing and jet along configurations to serve as baseline cases. Typical aerodynamic data from the interaction case are then compared to the baseline cases.

Figure 6 compares the pressure coefficients on the wing surfaces for the mid-span plane (above the jet centerline) for the clean wing and the interaction cases, results obtain from XFoil for the two-dimensional aerofoil are also listed. It can be seen that jet flow changes the wing surface pressure distributions, as a results reduces the lift generated by the wing. The lift coefficient drops from 0.21 to 0.13 . Further away from the jet it recovers to the clean wing level as the influence of jet is weakened.

The high speed jet reduces the pressure coefficient on the wing lower surface especially near the leading edge, and also increases the values on wing upper surface. The maximum $C_{p}$ reduces from 1 for the clean wing case at the leading edge stagnation point to 0.8 in the interaction case. A high pressure spot is also found on the top side of the jet nozzle underneath the wing.

This is because the characteristics of flow around the wing is changed under the influence of the high speed jet. For the geometry under consideration separation is found on both sides of the wing. However the flow on the pressure side reattaches further downstream, forming a recirculation zone on the wing surface. Influence of the jet stream reduces the recirculation zone on the lower surface, which is cleanly seen in Fig. 7 and Fig. 8. Streamlines are plotted for the two cases using velocity information on the first layer off the solid wall. The variation in the attachment points in the spanwise direction for the interaction case also indicates the influence of the jet on flow recirculation zone.

The narrow gap between the wing and the nozzle generates a suction effect (Coanda effect). The flight stream from both sides goes around the nozzle in a helical fashion and is drawn towards the top of the nozzle, creating a region of high pressure region, while on the bottom side of the nozzle, two low pressure regions appear at the centre of the two small circulation region, as can be seen in Fig. 8. Therefore the nozzle surface contributes a negative lift.

The jet centerline velocity experiences a sudden increase after leaving the nozzle to a Mach number of 0.77 within $0.5 D_{j}$, as is shown in Fig. 9(a) for the streamwise velocity along the jet axis. This is due to the particular nozzle used. There is a short divergence section at the nozzle exit. The centerline velocity 
gradually recovers to $\mathrm{M}=0.75$ within four jet exit diameter, near the end of the potential core, which is $4.8 D_{j}$ long. Analysis of the baseline case showed that the decay rate of jet centerline velocity is in good agreement with analytical results and self-similarity is achieved further downstream.

Under the influence of the wing jet centerline velocity decreases faster compared to the baseline case due to the blockage effect of the wing, which overlaps in the streamwise direction with the nozzle. The jet axial velocity profile becomes asymmetric. The expansion of the jet is restricted by the wing. Profiles of mean velocity in cross-flow planes at different streamwise locations $\left(x / D_{j}=1,2,3\right.$ and 5 downstream of the nozzle exit are plotted in Fig. 9(b), which clearly visualizes the asymmetric nature of the jet around the geometric nozzle centerline.

Another interesting feature to note is that the jet stream is shifted towards the wing as it shown in Fig. 10 for the mean axial velocity contours in a x-z plane across jet centerline. It can be seen that center of the profile is shifted towards the wing. This is due to the low pressure region appeared underneath the wing, which can be seen from wing surface pressure contours (not shown here). The shifting angle for the current configuration is about one degree.

Contours of the second invariant of the velocity gradient is shown in Fig 11. It can be seen that current LES is able to resolve the flow structures to very fine details. The flow around the wing is mainly twodimensional near the leading edge and long spanwise structures are found underneath the wing. Further downstream, under the influence of the jet, three-dimensional flow features are developed. More turbulent structures are seen for shear layer near the wing and they interact with the wing which causes surface pressure fluctuations, and in turn, generate noise. The vortical structures in the jet region introduce strong mixing between the jet and the flight streams. Interaction between vortical structures is the main noise sources from a jet ${ }^{18}$. Analysis showed that the interaction between jet and the clean wing does not happen until near the wing trailing edge. The main jet stream does hit the wing.

\section{B. Jet interacting with a flap-deployed wing}

Similar to the jet/clean wing case, simulations were performed for the wing with flap deployed as the baseline case and for jet interacting with the flap-deployed wing. Comparisons were then made between these two cases and the jet along case to identify the installation effects when the flap is deployed.

Figure 12 depicts contours of the instantaneous axial velocity in a $x-z$ plane along the jet axis at $y=0$ for jet interacting with the flap-deployed wing. Compared to the clean wing, wing with deployed flap and jet/clean wing interaction cases, much stronger vortical structures are seen in this case. The jet interacts strongly with the wing. The wing is wetted by the jet stream in the flap area. Contours of the second invariant of velocity gradient are plotted in Fig. 13 showing the structure around the wing and downstream of the jet. It is obvious that the vortical flow from the jet interacts with the wing flap. Analysis of the velocity field (not shown here) showed that the jet stream is first sucked toward the main element of the wing by a very small angle (less than one degree) and then deflected away from the wing.

A detailed flow field in a region around the main element trailing edge and the flap is shown in Fig. 14 as streamline plots. It can be seen that the jet stream increases the recirculation zone in the flap cover and moves the stagnation point from the flap leading edge further downstream on to the lower surface, which results in an increasing in circulation around the flap and the lift contribution from the flap. The jet stream fits the flap and is deflected downwards.

When the flap is deployed the flow separates on the upper surface of the flap, forming a large separation zone behind the flap. Under the influence of the jet the flow on the upper surface is reattached on to the flap, leaving a small recirculation zone on the flap top surface. This changes the pressure distribution on the wing surfaces and causes a change in the pressure coefficient and lift, as shown in Figure 15.

Further analysis of the flow field will be carried out to clarify the change to the flow dynamics and to quantify the effects of jet interacting with the flap-deployed wing.

\section{Noise prediction}

Flow field data are collected on different FW-H integration surfaces every 100 time steps over a period equivalent to 330,000 time steps for the first three cases. Based on the grid resolution around our control surface and assuming that with our numerical schemes that 8 points per wavelength are needed to accurately resolve an acoustic wave, the maximum frequency resolved is $4 \mathrm{kHz}$. The far-field radiated sound can be 
calculated using three types of FW-H surfaces,

- On the solid surfaces

- Off-body closed surface

- Off-body open surface

The complete three-dimensional integration surfaces are illustrated in Fig. 16(a), where the instantaneous pressures are also plotted on those three integration surfaces.

Initial attends have been made to use the source data for farfield predictions. Farfield radiated sound is calculated using all three FW-H integration surfaces, and are shown in Fig. 16(b) for the case of jet interacting with a clean wing. It is clear that for the jet/wing interaction case, the on-body integration surface, which only includes the wing surface, has not considered the sources in the jet region, thus is not adequate to investigate the installation effect. It also shows that the contribution to far field sound from wing surface source is much smaller than contributions from jet stream.

Simulations have been run for all five configurations. Acoustic source data have been collected for the three cases (jet along, clean wing, and jet interacting a clean wing), and are collecting more data for the other two cases (wing with flap deployed and jet interacting with flap-deployed wing). The aeroacoustic impact of the installation effect will be studied in detail by comparing acoustic results from all five cases.

\section{Conclusions}

The main focus of this work is to use time-accurate numerical tools to investigate the interaction of the vortical flow from jet engine with high-lift devices. Large-eddy simulations have been performed for turbulent flow around aircraft wing, with and without the influence from the jet. Simulations were undertaken for five different geometries, namely wing alone, jet alone, jet/clean wing, wing with deployed flap and jet/wing with deployed flap. Large amount of data have been obtained from the simulations, initial analysis of the results showed that

- When the nozzle is under a clean wing the jet stream does not hit the wing, however when the flap is deployed the jet stream wets the wing flap.

- For the jet/clean wing interaction case, the jet stream is shifted towards the wing by a small angle (one degree) due to the low pressure region, which is in turn caused by the high speed jet, appeared under the wing. However when the flap is deployed the jet stream is shifted slightly towards the wing main element and then is deflected away from the wing by the flap. The lift from the main element is reduced however

- The interaction reduces lift for the clean wing. However when the flap is deployed the reduction in lift contribution from the main element is compensated by an increase from the flap.

- Acoustic source data have been collected from LES results for three cases: jet, wing and jet/clean wing interaction cases, and are collecting more data for the two cases with deployed flap. Comparison of aeroacoustic results with experimental measurements will be presented in future publications.

\section{Acknowledgements}

This study is performed under WP 3.2 of SYMPHONY, a project supported by Technology Strategy Board, UK. Supports from industrial partners Airbus, Rolls-Royce, of the project are greatly appreciated. Experiments are performed by project partners in QinetiQ.

\section{Reference}

1. Way, D.J. \& Turner, B.A. 1980 Model tests demonstrating under-wing installation effects on engine exhaust noise. AIAA Paper 80-1048.

2. Wang, M.E. 1980 Wing effect on jet noise propagation. AIAA Paper 801047. 
3. Reddy, N.N., \& Tanna, H.K. 1980 Installation effects on jet noise in flight. AIAA Paper 801044.

4. Stevens, R.C.K., Bryce, W.D. \& Szewczyk, V.M. 1983 Model and full-scale studies of the exhaust noise from a bypass engine in flight. AIAA Paper 830751.

5. Mead, C.J. \& Strange, P.J.R. 1998 Under-wing installation effects on jet noise at sideline. AIAA Paper 982208 .

6. Bhat, T.R.S. 1998 Jet-flap installation noise. NASA Contractor Final Report on Contract NAS 120267, Task 17, Subtask 2.

7. Hixon, R., 2000 Prefactored small-stencil compact schemes, Journal of Computational Physics.

8. Ashcroft, G., \& Zhang, X., 2003 Optimized Prefactored Compact Schemes," Journal of Computational Physics.

9. Hu, F. Q., Hussaini, M. Y., \& Manthey, J, 1996 Low-dissipation and -dispersion Runge-Kutta schemes for computational acoustics," Journal of Computational Physics, 124, 177-191.

10. Jameson, A., 1991 Time Dependent Calculations using Multigrid with Applications to Unsteady Flows Past Airfoils and Wings, AIAA Paper 91-1956.

11. Ma, Z. K., Zhang, X., Smith, M. G., Molin, N., \& Chow, L. C., 2007 Broadband Slat Noise Attenuation Potential with Acoustic Liner Treatment," AIAA Paper.

12. Kim, J. W., \& Lee, D. J., 2003 Characteristic Interface Conditions for Multiblock High-Order Computation on Singular Structured Grid," AIAA Journal, Vol. 41, pp. 2341-2348.

13. Spalart, P., \& Allmaras, S., 1992 A One Equation Turbulence Model for Aerodynamic Flows, AIAA Paper, 92-0439.

14. Piomelli, U., \& Chasnov, J. R., 1996 Large-Eddy Simulations: Theory and Applications, Transition and Turbulence Modelling.

15. Hedges, L. S., Travin, A. K., \& Spalart, P. R., 2002 Detached-Eddy Simulations over a Simplified Landing Gear," Fluid Engineering, Vol. 124.

16. Chen, X., Huang, X. \& Zhang, X. 2009 Sound radiation from a bypass duct with bifurcations, AIAA Journal, 47(2), 429-436.

17. Peers, E.; Zhang, X. \& Kim, J. W., 2010 Patched characteristic interface condition for high-order multiblock aeroacoustic computation, AIAA Journal, 48(11), 2512-2522, DOI: 10.2514/1.J050130.

18. Grizzi, S. \& Camussi, R., 2012 Wavelet analysis of near-field pressure fluctuations generated by a subsonic jet, Journal of Fluid Mechanics, Vol. 698, 93-124. 


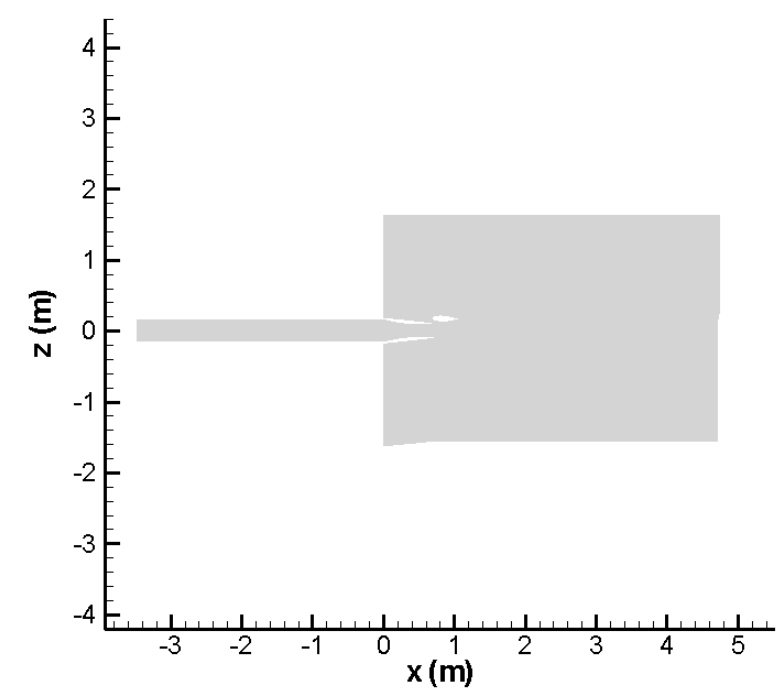

Figure 1: Computational domain and sketch of the wing/nozzle model.

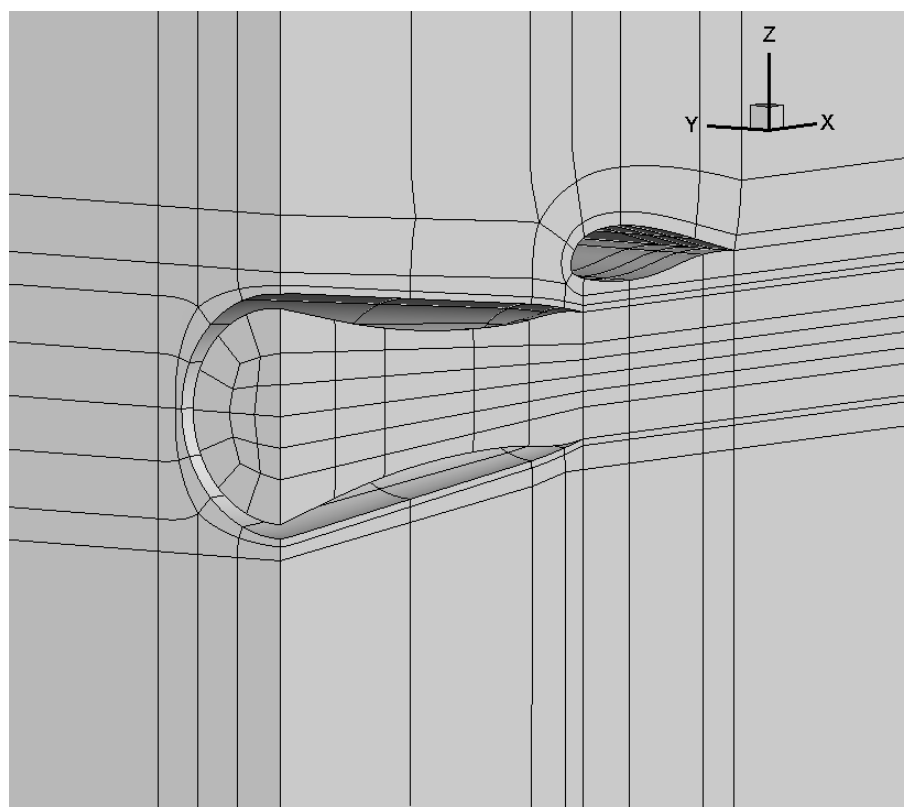

Figure 2: Topology of the fully-structured grid for the jet/clean wing case. 


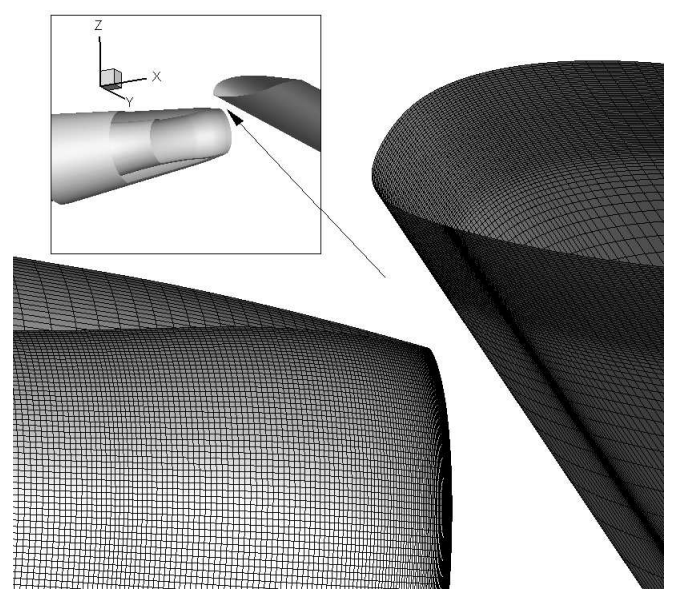

(a)

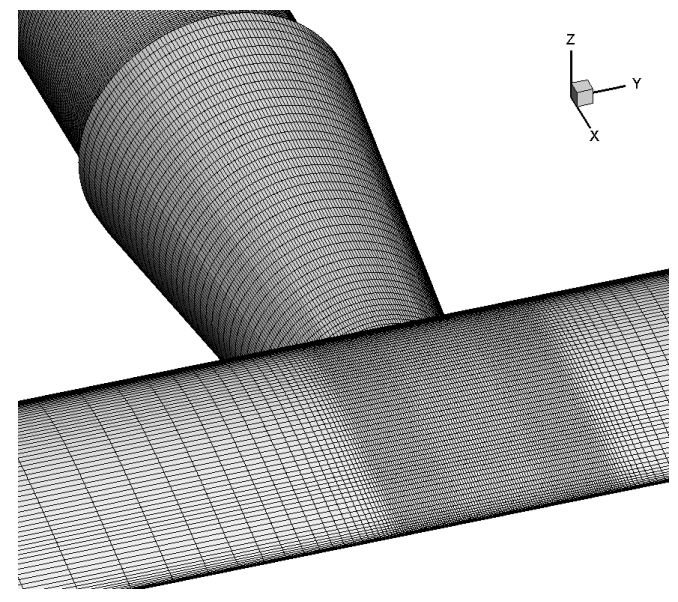

(b)

Figure 3: Close-up of the jet/clean wing grid, view from top, show for every other grid points.

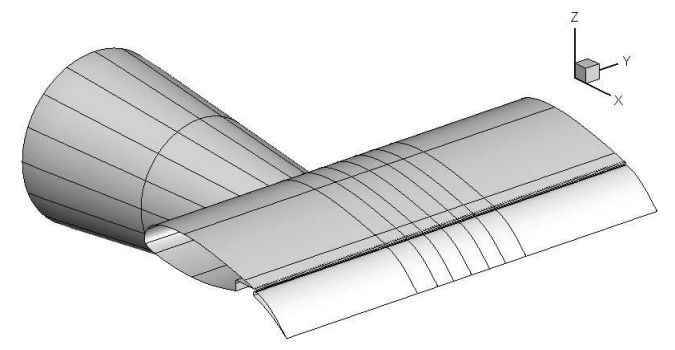

(a) View from top

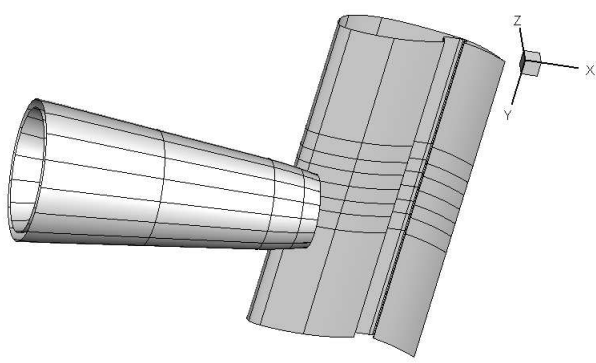

(b) View from bottom

Figure 4: Mesh topology for the jet/flap-deployed wing interaction case.

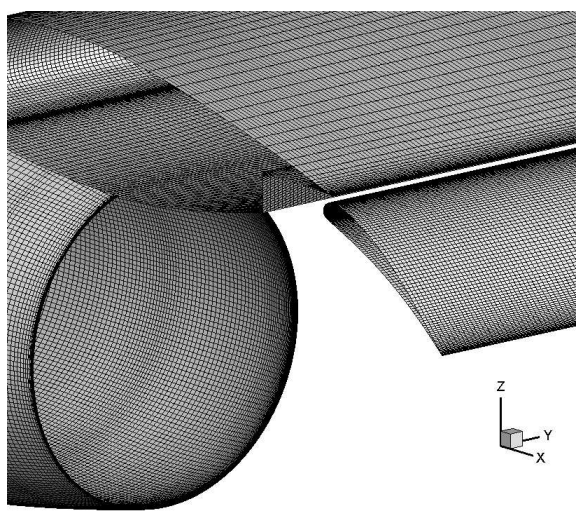

(a) View from top

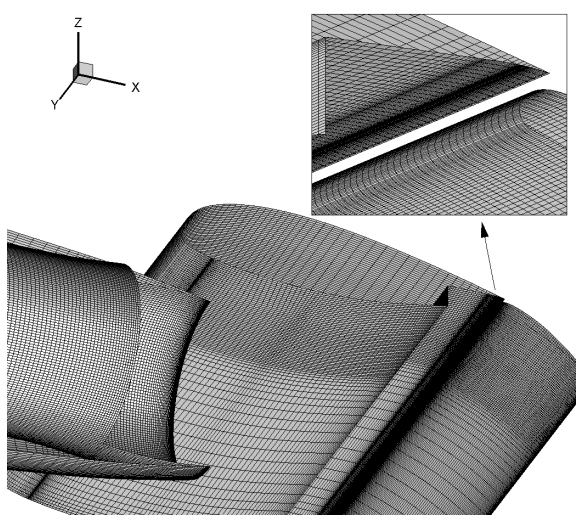

(b) View from bottom

Figure 5: Close-up of grid for the jet/flap-deployed wing interaction case. 


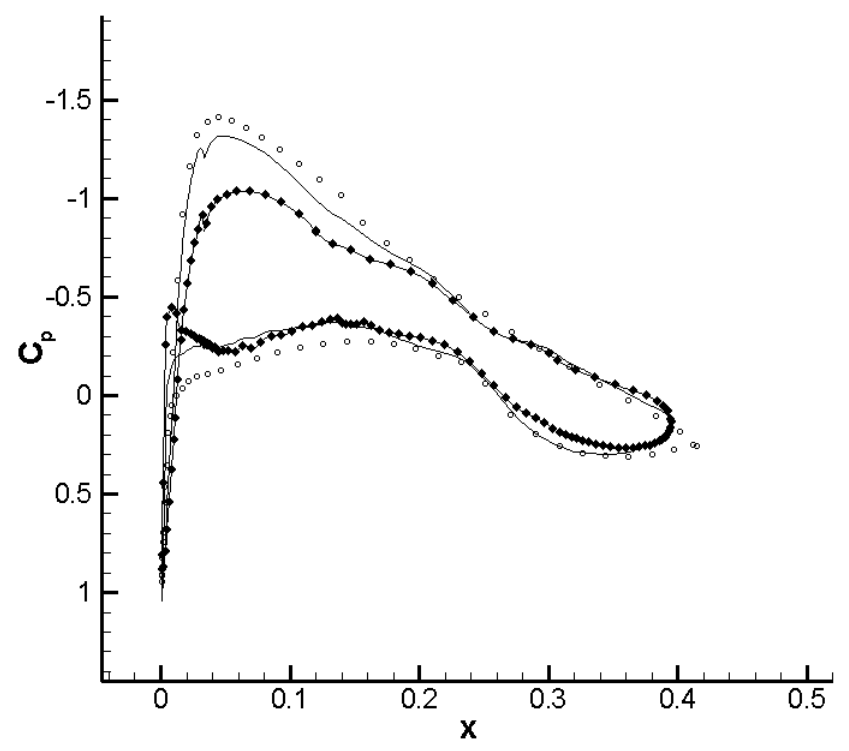

Figure 6: Profiles of pressure coefficients on the wing surface. Wing LES (Solid line); Jet/wing (diamond); Wing XFoil (circle).

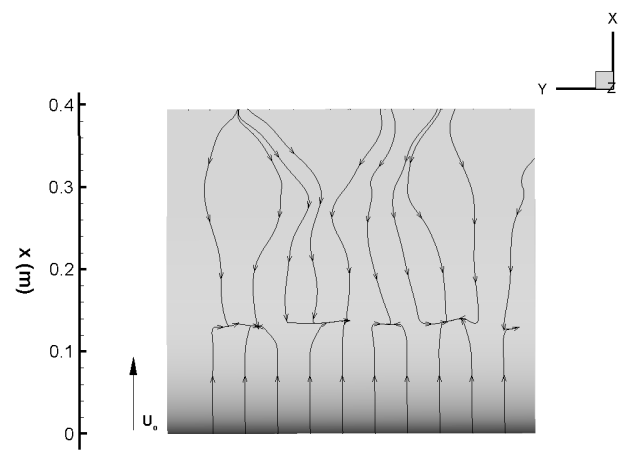

(a) View from top

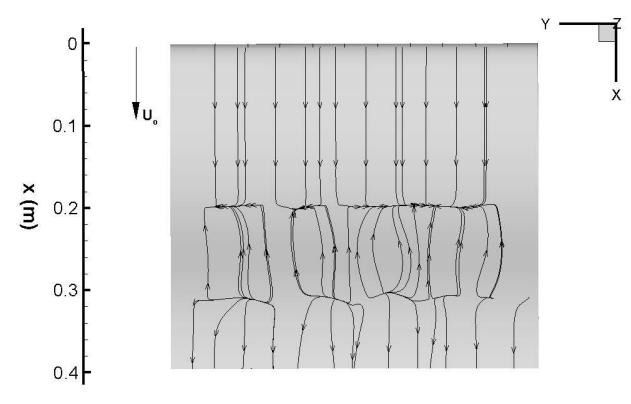

(b) View from bottom

Figure 7: Streamlines for flow near the surface of the wing for the clean wing case. 


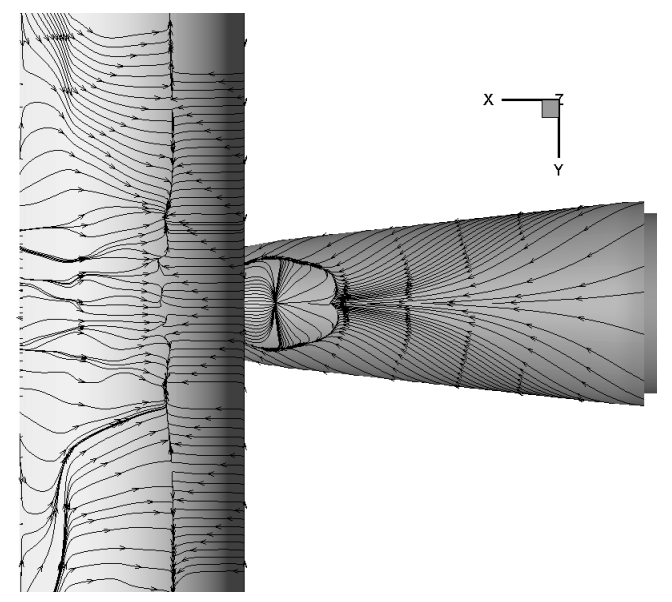

(a) View from top

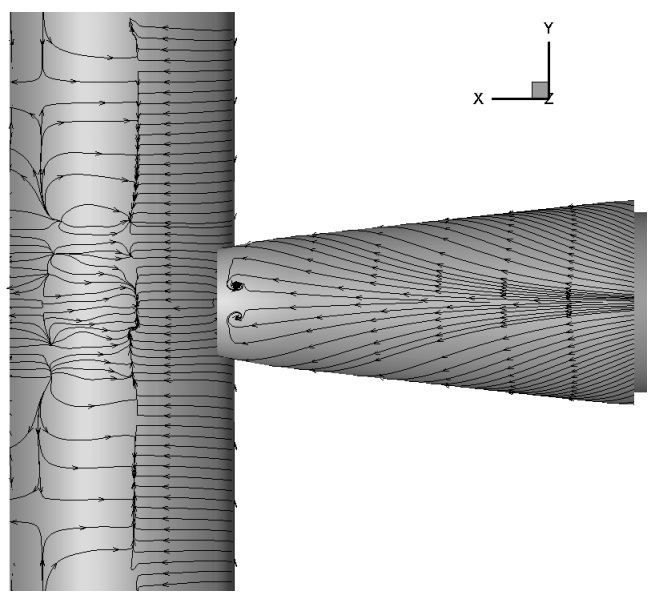

(b) View from bottom

Figure 8: Streamlines for flow near the surface of the wing and nozzle for the jet/clean wing interaction case.

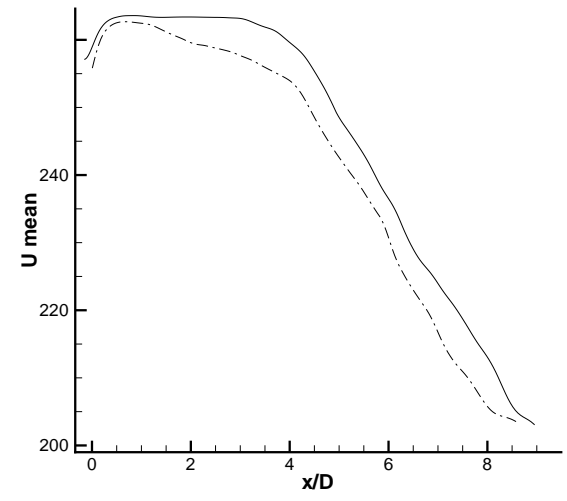

(a) Velocity profiles along the centerline. Solid line: jet along; Dash line: jet/clean wing.

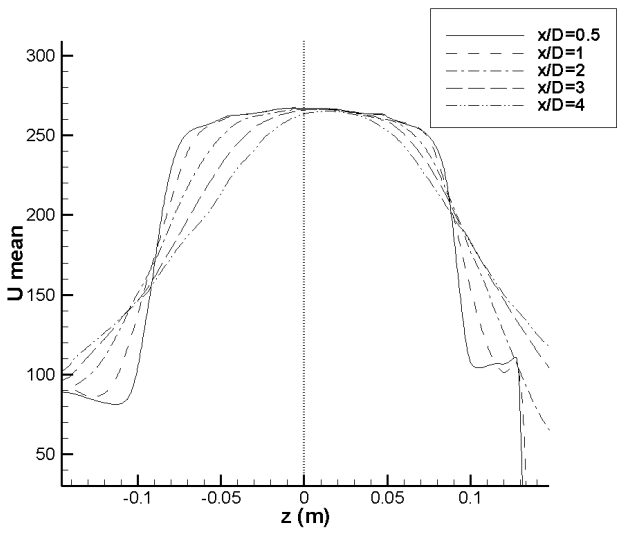

(b) Velocity profiles at different locations for the jet/clean wing interaction case.

Figure 9: Velocity streamwise velocity profiles.

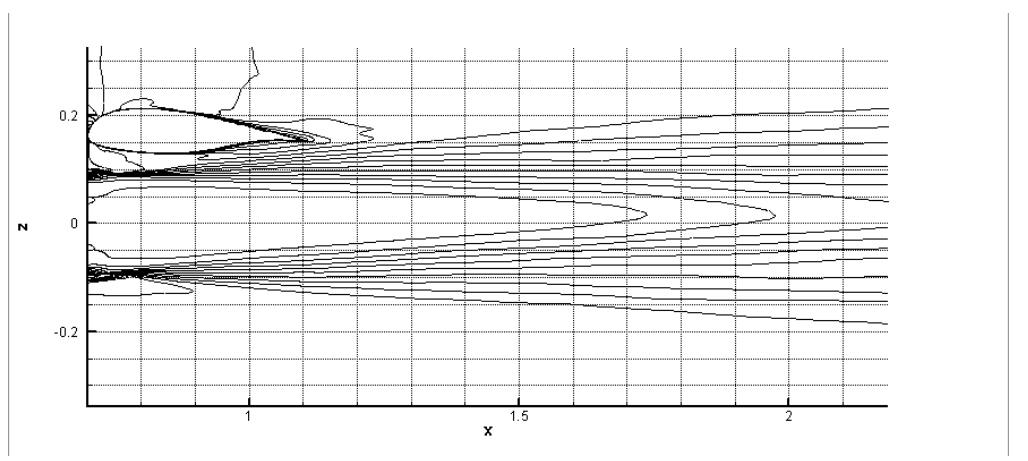

Figure 10: Contour of mean streamwise velocity in x-z plane along jet centerline. 


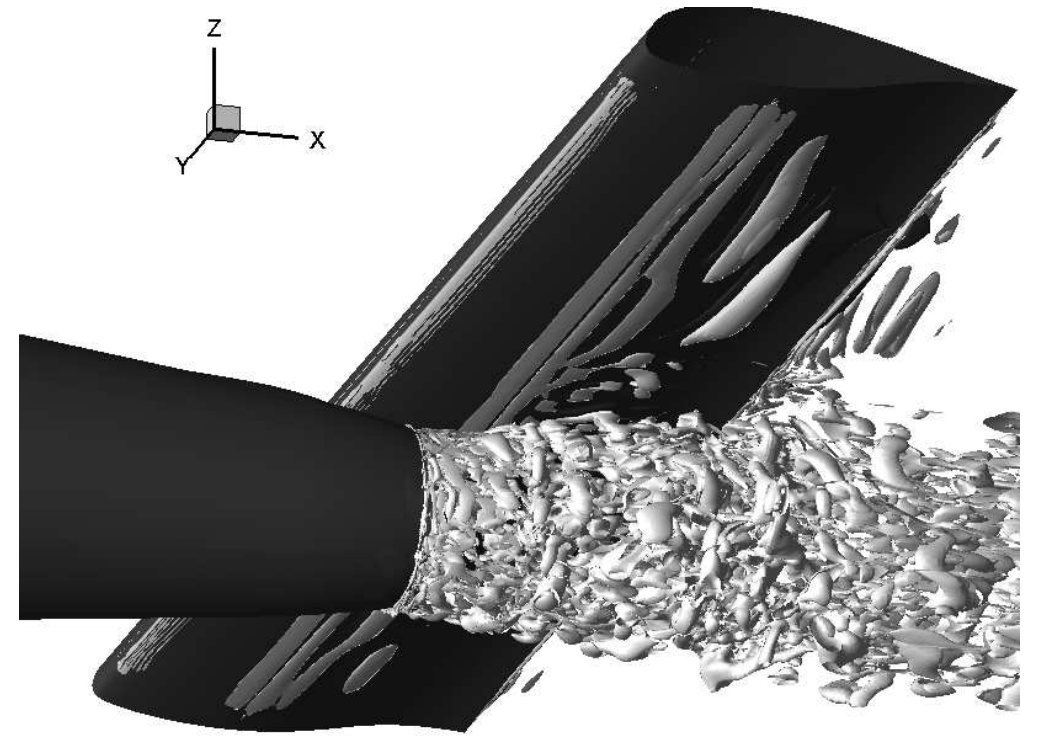

Figure 11: Iso-surface of $\mathrm{Q}$, the second invariant of velocity gradient tensor.

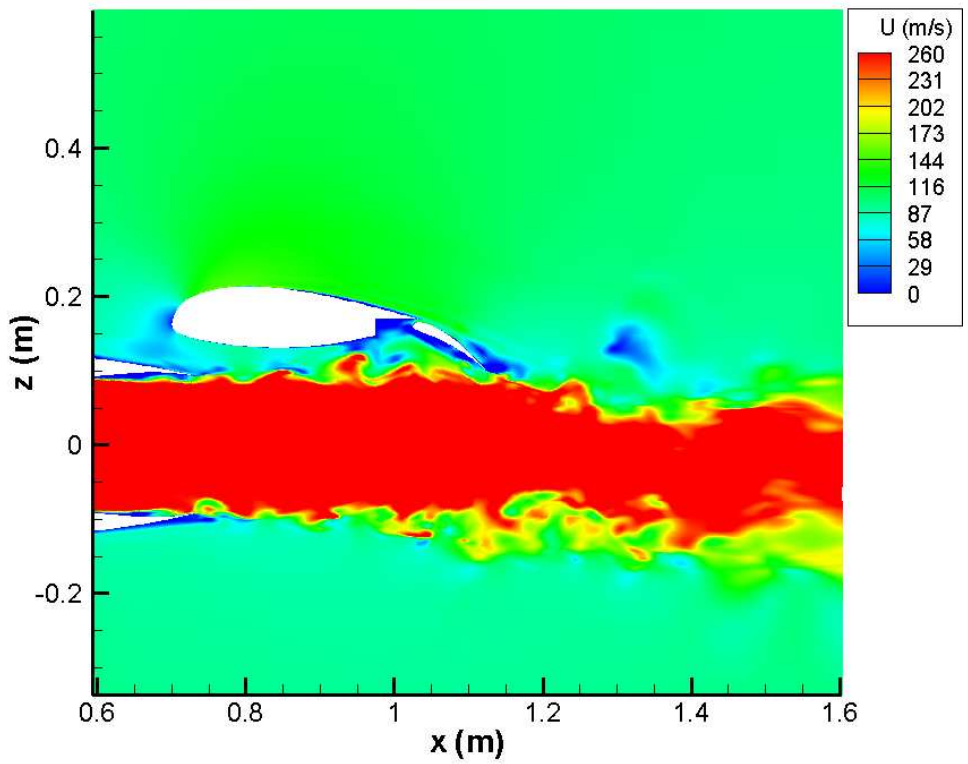

Figure 12: Contours of instantaneous streamwise velocity in the wing mid-span plane. 


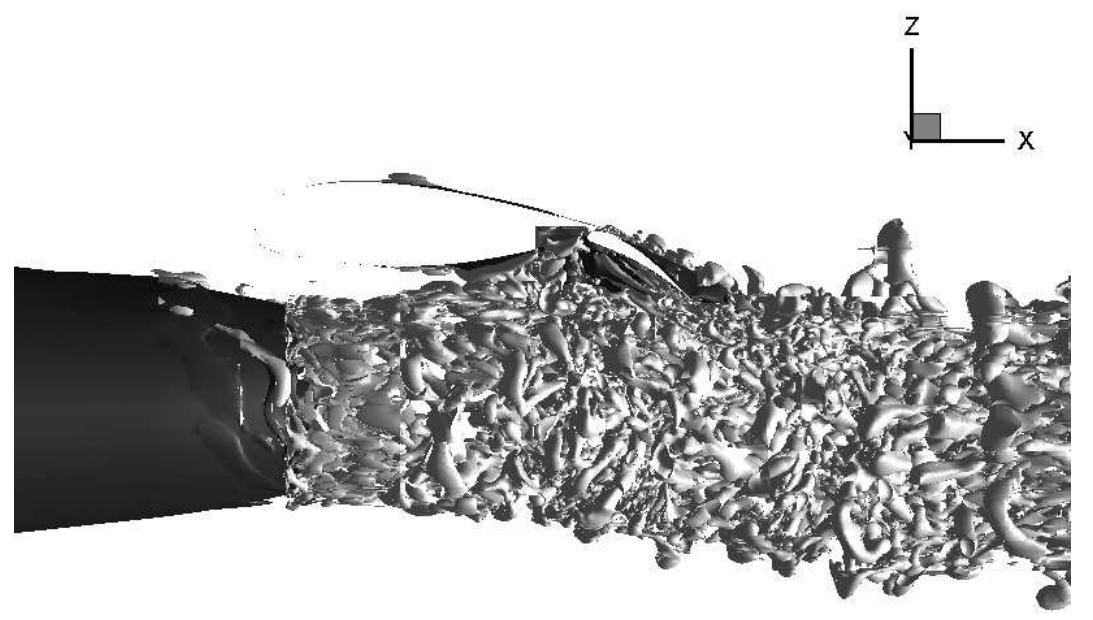

(a) Side view

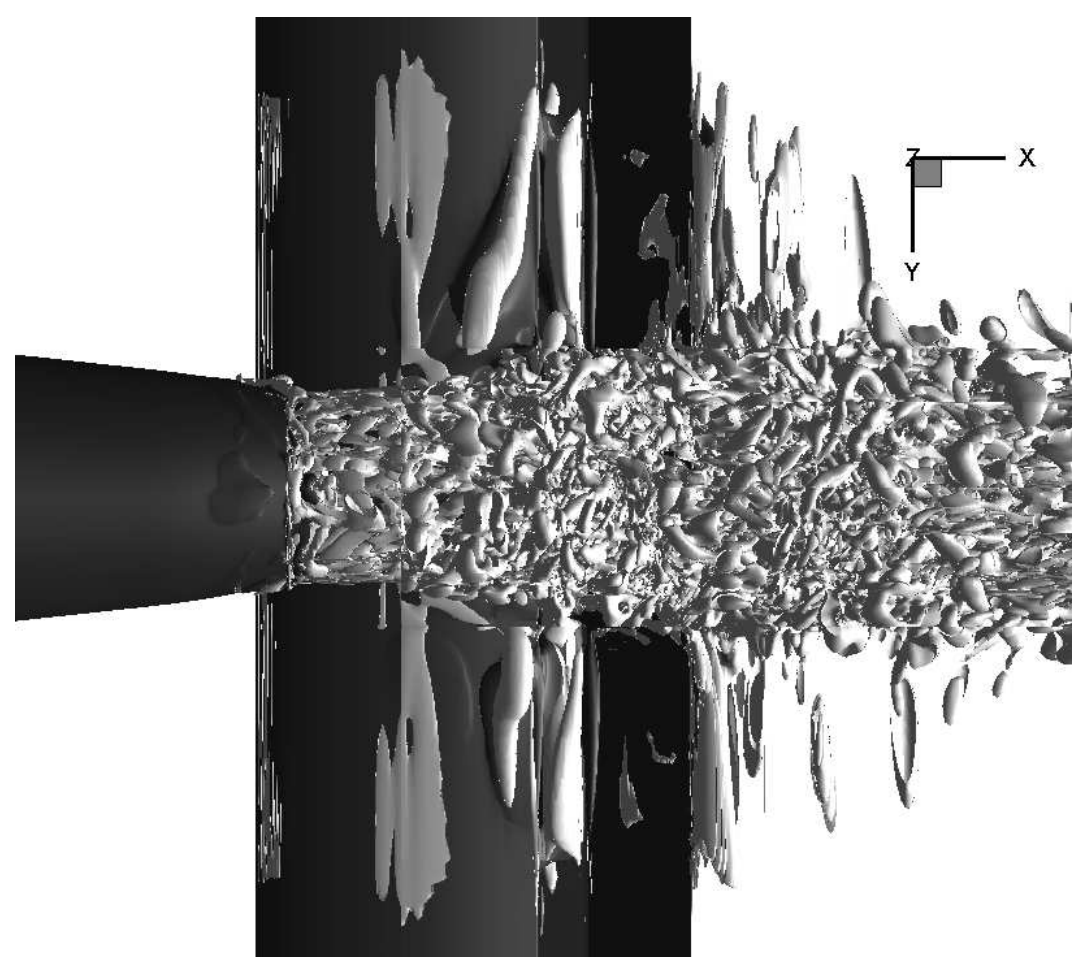

(b) bottom view

Figure 13: Iso-surface of the second invariant of velocity gradient for the jet/flap-deployed wing interaction case. 


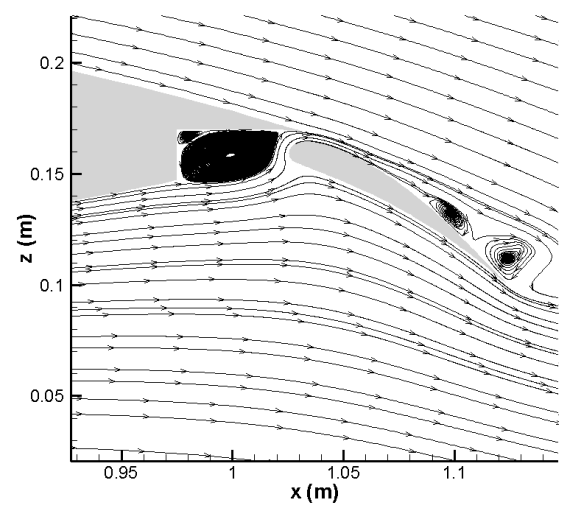

(a) Without jet

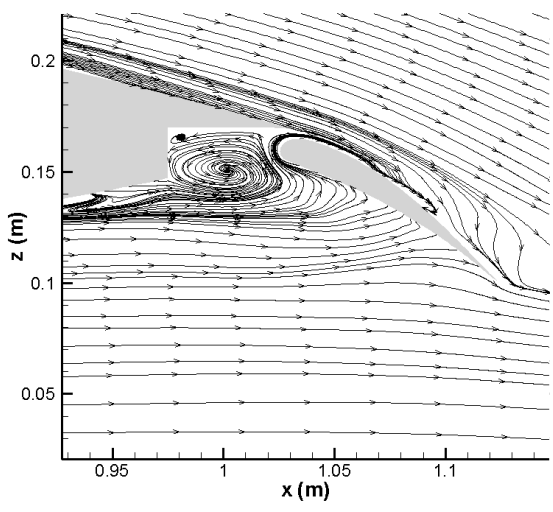

(b) With jet

Figure 14: Streamlines for flow around the deployed flap.

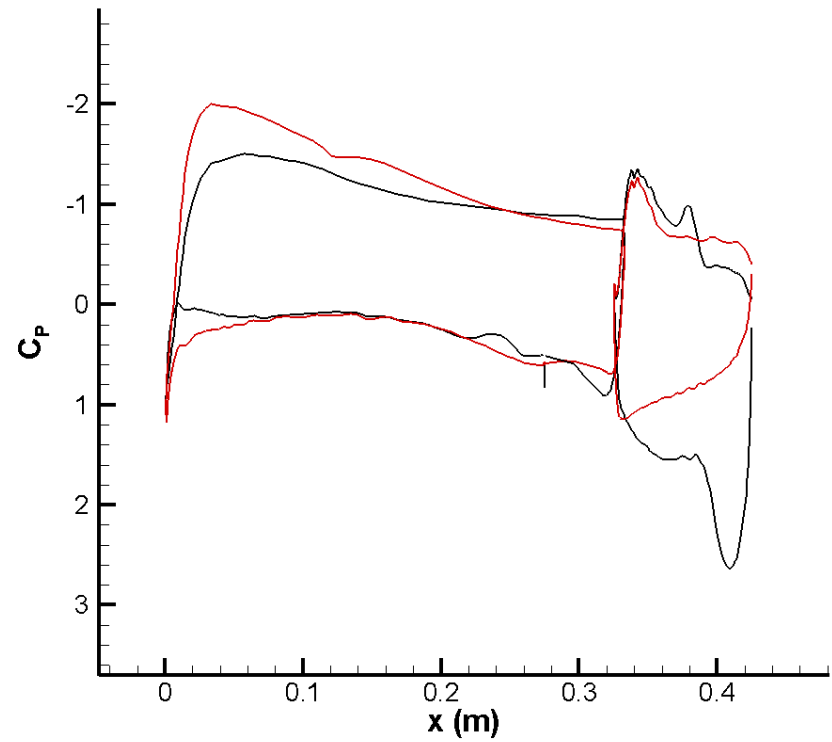

Figure 15: Comparison of $C_{p}$ profiles for deployed wing cases with jet (Black) and without jet (Red). 


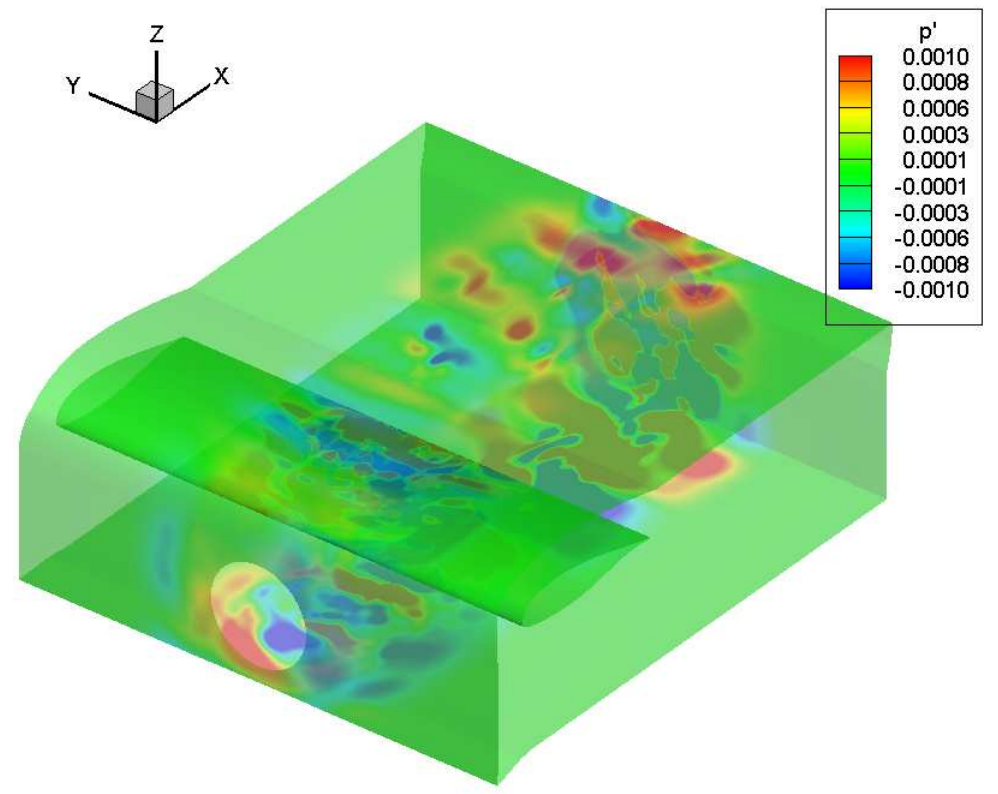

(a) Instantaneous non-dimensional pressure on a $\mathrm{FW}-\mathrm{H}$ integration surface

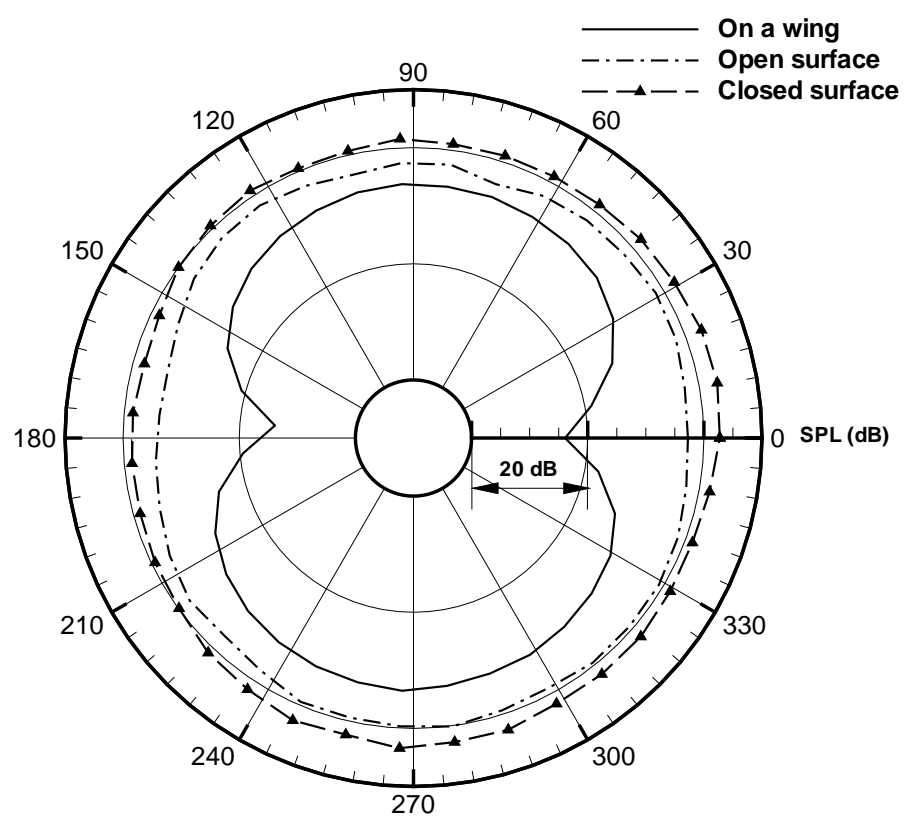

(b) SPL from different FW-H surfaces for observers $10 \mathrm{~m}$ from the jet exit

Figure 16: Farfield directivities from different integration surfaces for jet interacting with a clean wing. 